
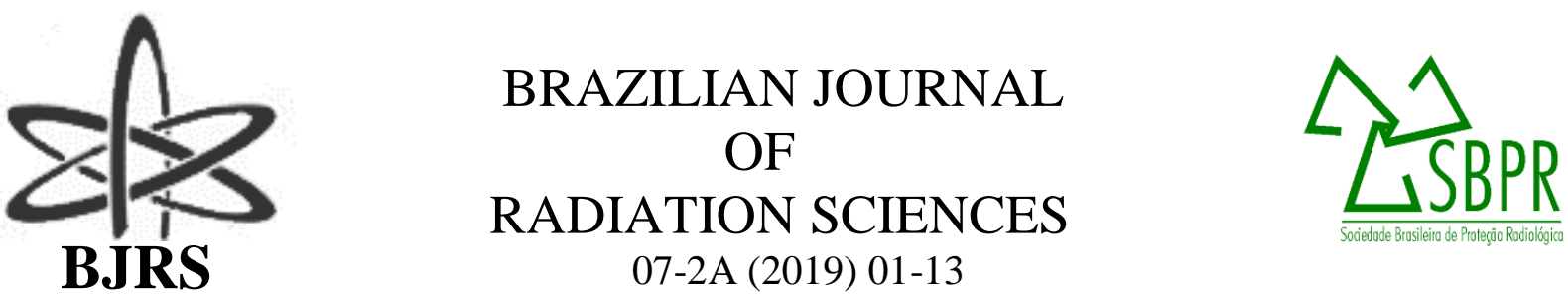

\title{
Image quality assessment using NEMA NU 4/2008 standards in small animal PET scanner
}

\author{
R. M. G. Gontijo ${ }^{a, b}$; A. V. Ferreira ${ }^{a}$ J. B. Silva ${ }^{a}$; M. Mamede ${ }^{a, b}$ \\ ${ }^{a}$ Radiopharmaceuticals Research and Production Unit, Center of Nuclear Technology Development, 31270-901, \\ Belo Horizonte-MG, Brasil. \\ ${ }^{b}$ Department of Anatomy and Imaging, Federal University of Minas Gerais, 30130-100, Belo Horizonte-MG, Brasil. \\ rodrigo.gontijo@cdtn.br
}

\begin{abstract}
In Brazil, there are few microPET in use and a quality control protocols standardization are needed to harmonize their use in the research field. Thus, the purpose of this study is to characterize the image quality performance of the microPET scanner (LabPET 4, GE healthcare Technologies, Waukesha, WI) using the NEMA NU 4/ 2008 standards and specific phantom. The NEMA image-quality (IQ) phantom consists of 3 different regions to analyze distinct characteristics: image noise (\%SD), expressed as percentage SD in a uniform region (\%SD), recovery coefficient (RC) and Spill-over (SOR) in air and water. The IQ phantom was filled with 18F-FDG calibrated at the beginning of acquisition, placed in the center of the field-of-view (FOV) and measured with the typical whole body imaging protocol. The images were reconstructed with different reconstruction methods (FBP-2D; MLEM-3D and OSEM-3D); with and without high resolution (HR) when possible. The results were compared. The LabPET 4 system produces appropriate image and with performance according to the literature. The present study is an initial step to verify the NEMA NU 4/2008 use in the Brazilian scenario for further standardization.
\end{abstract}

Keywords: Image Quality, Positron Emission Tomography, NEMA.

ISSN: 2319-0612

Accepted: 2018-12-03 


\section{INTRODUCTION}

The importance of small-animal positron emission tomography (microPET) has increased during the last decades as a valuable tool for studying animal models of human disease and to contribute in the development of new radiopharmaceuticals or in studies of new applications of traditional radiopharmaceuticals [1]. Because of widespread use and commercial availability of microPET scanners, the National Electrical Manufactures Association (NEMA) published its NU 4/2008 standards [2], a consistent and standardized methodology for measuring scanner performance parameters for smallanimal PET imaging.

In Brazil, there are few microPET in use and a standardization of quality control protocols is needed to harmonize their use in the research field. Thereby, the purpose of this study is to characterize the image quality performance of the microPET scanner (LabPET 4, GE Healthcare Technologies, Waukesha, WI) using the NEMA NU 4/ 2008 standards and specific phantom.

\section{MATERIALS AND METHODS}

All experiments were carried out at CDTN/CNEN in the Molecular Imaging Laboratory (LIM) and with collaboration of the Radiopharmaceutical Research and Production Unit (UPPR), which made the $18 \mathrm{~F}-\mathrm{FDG}$ sources available.

\subsection{System description}

The Triumph ${ }^{\mathrm{TM}}$ platform is a preclinical system dedicated for rodents imaging. The subsystem LabPET 4 consists of a stationary gantry with 1536 detectors. It employs an Avalanche Photo Diode (APD) detector ring incorporating an assembly of LYSO (Lutetium yttrium oxyorthosilicate Lu1.9Y0.1SiO5) and LGSO (Lutetium gadolinium oxyorthosilicate - Lu0.4Gd1.6SiO5) scintillators optically coupled one after the other [3]. 
LabPET 4 images are acquired using a 250-650 keV energy window and $22 \mathrm{~ns}$ coincidence timing window. It provides axial field of view (FOV) of $3.7 \mathrm{~cm}$ and can operate in a dynamic or static mode. Coincident data are saved in list mode and can be sorted out as sinograms. Some important features are compiled in Table 1. More details about the LabPET 4 design and architecture are presented elsewhere $[4,5]$.

Table 1: Summary of the LabPET $4^{\mathrm{TM}}$ technical specifications.

\begin{tabular}{ll}
\hline \multicolumn{1}{c}{ Parameter } & \multicolumn{1}{c}{ Specification } \\
\hline Detector Crystal Material & Phoswich pair of LYSO and LGSO \\
Crystal Dimension & $2.0 \times 2.0 \times 14 \mathrm{~mm} 3$ \\
$\mathrm{~N}^{\mathrm{o}}$ of detector rings & 24 \\
Crystals per ring & 64 \\
$\mathrm{~N}^{\mathrm{o}}$ Total of Crystals & 1536 \\
Axial field-of-view & $37 \mathrm{~mm}$ \\
\hline
\end{tabular}

The reconstruction algorithms available in LabPET 4 (CDTN/CNEN) are filtered backprojection (FBP), maximum likelihood expectation maximization (MLEM) and ordered subset expectation maximization (OSEM). The system has also the option of image reconstruction in high resolution mode using $0.25 \mathrm{~mm}$ pixel size instead of $0.5 \mathrm{~mm}$ used in ordinary mode.

\subsection{Image Quality Phantom}

Section 6 of the NEMA 4 purposes to produce images that simulate those acquired in the whole body study of a small animal. For this purpose, a specially designed NEMA NU 4 - 2008 image quality (IQ) phantom made up of polymethylmethacrylate (PMMA) was used. Figure 1 presents a schematic view of the IQ phantom and Figure 2 illustrates the schematic coronal and axial section views of phantom. 
Figure 1: Micro-PET IQ phantom representation.

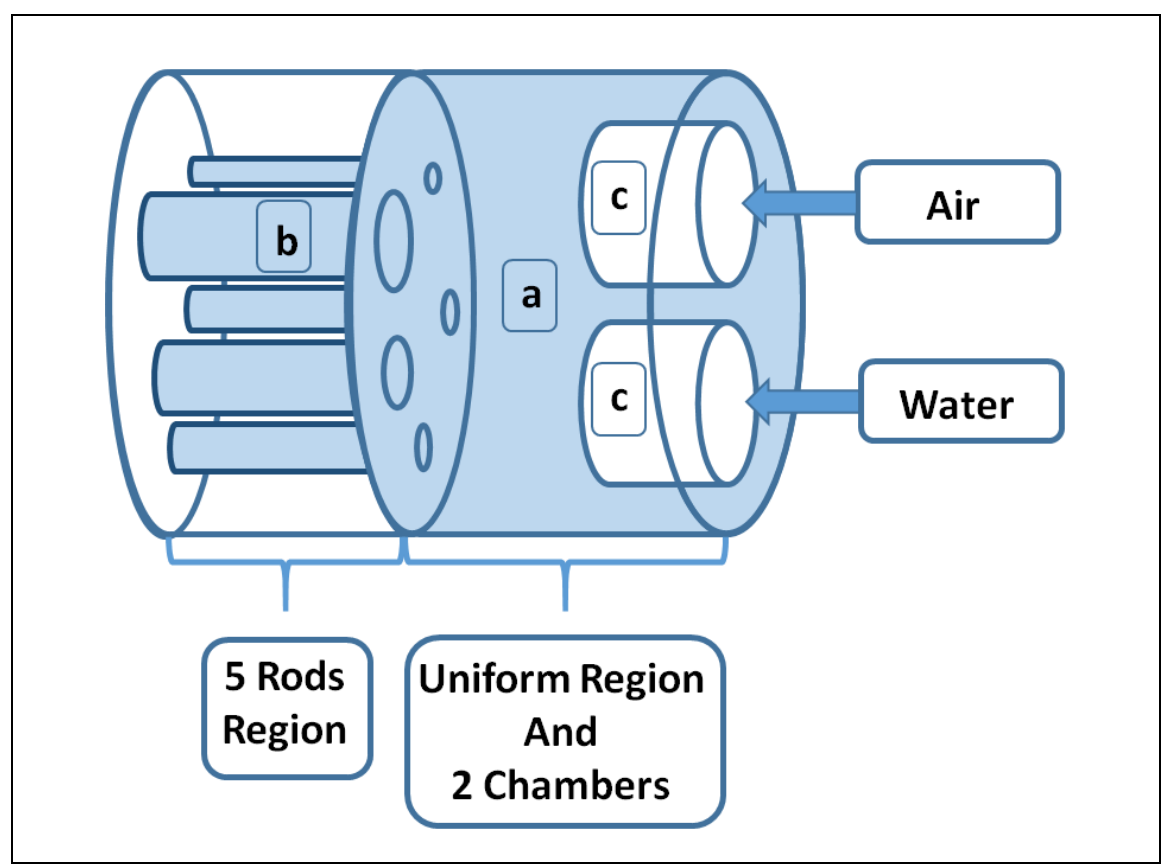

Figure 2: Schematic views of NEMA NU 4-2008 IQ phantom.

(A) Coronal-Section view and (B) Axial-Section view through rods region.

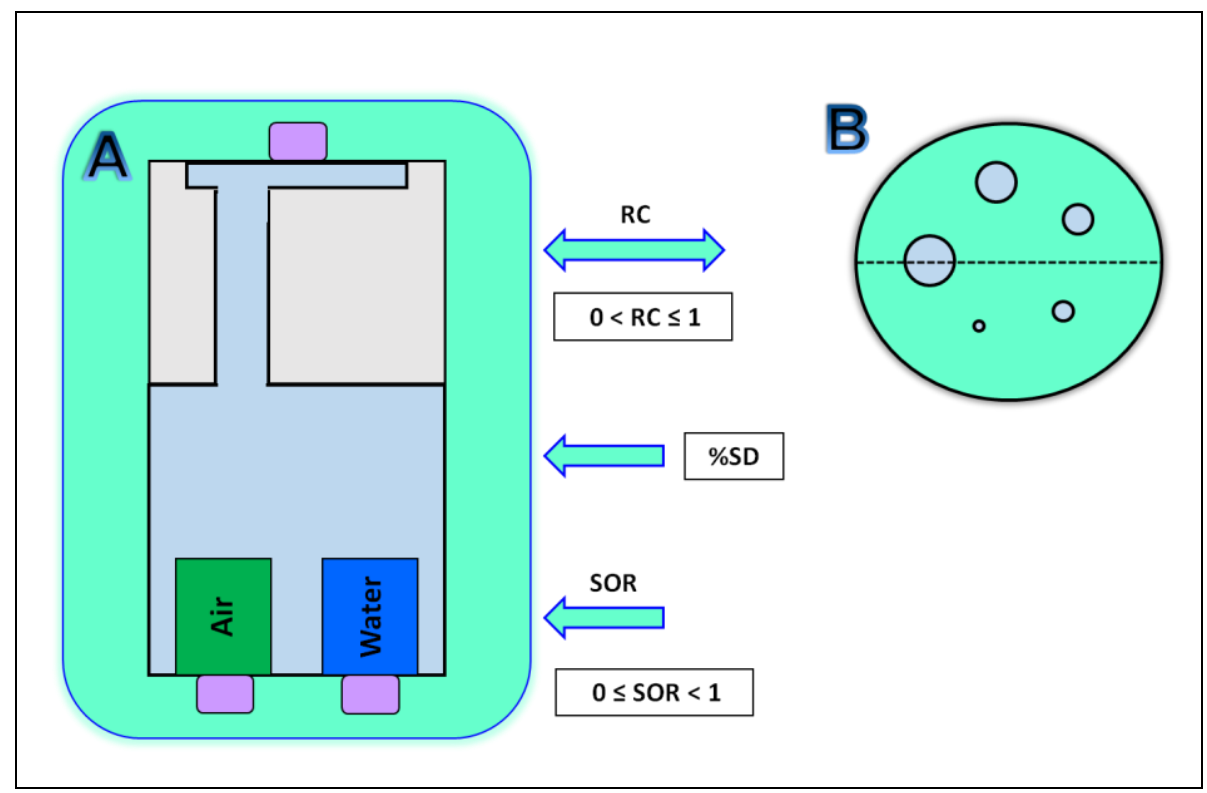

The IQ phantom consists of 3 distinct segments to analyze different aspects of image quality, discriminated in Table 2: 
Table 2: Image Quality Phantom Regions.

\begin{tabular}{ccc}
\hline & Regions & Representation \\
\hline $\mathbf{1}$ & 5 Rods of different diameters & $\mathrm{b}-$ in the Figure 1 \\
$\mathbf{2}$ & Uniform Region & $\mathrm{a}-$ in the Figure 1 \\
$\mathbf{3}$ & 2 non-radioactive chambers & $\mathrm{c}-$ in the Figure 1 \\
\hline
\end{tabular}

The first region (a) of the phantom body is solid, with 5 fillable rods with diameters of 1, 2, 3, 4 and $5 \mathrm{~mm}$. These are important to determine the recovery coefficient (RC), defined as the ratio between the measured activity concentration in the rods and the activity concentration measured in the uniform area [2].

The intermediate, uniform region (b) is used to determine the mean, maximum and minimum activity concentration and respective percentage standard deviation (\% $\mathrm{SD})$ as a measure of noise [2].

The third region (c) of the phantom body is a fillable cylindric chamber with 2 cylindrical compartments (14mm internal length; $8 \mathrm{~mm}$ inner diameter), one is filled with air and the other with nonradioactive water. These 2 cold compartments are used to determine the spillover ratio (SOR) in air and water, defined as the activity concentration in each cold compartment relative to the mean activity concentration in the hot background [2].

Both RC and SOR parameters are theorically limited between 1 and 0 , being that $0<\mathrm{RC} \leq 1$ and 0 $\leq \mathrm{SOR}<1[6]$.

\subsection{Acquisition Procedures}

The whole IQ phantom, except cold chambers, was filled with $3.7 \mathrm{MBq}(100 \mu \mathrm{Ci})$ of $18 \mathrm{~F}-\mathrm{FDG}$ (at the start of the scan), for a 20 minutes acquisition. It was placed in the center of the FOV and measured with the typical whole body imaging protocol used in LIM/CDTN.

Before measurement, the phantom was cleaned and checked for any activity remaining from previous experiment. Normalization and random corrections were applied to the acquired data before image reconstruction. 
Images were reconstructed with three different reconstruction methods (FBP-2D; MLEM-3D and OSEM-3D); with and without high resolution (HR) when it is possible. The iterative method MLEM was applied using 20 iterations, while OSEM used 20 iterations and 4 subsets.

The treatment and analysis of the images were performed, as recommended in the NEMA NU 42008, using molecular imaging softwares AMIDE and PMOD.

\section{RESULTS AND DISCUSSION}

Results for uniformity, values of recovery coefficients and spillover ratio are presented in the sequence.

\subsection{Uniformity}

Figure 3 presents typical images of uniformity region and respective line profiles.

Figure 3: Transverse plane image of the uniform region (upper) and line profile across the uniform region of the IQ phantom (bottom). (A) AMIDE software; (B) PMOD software with a $3 D$ graph.

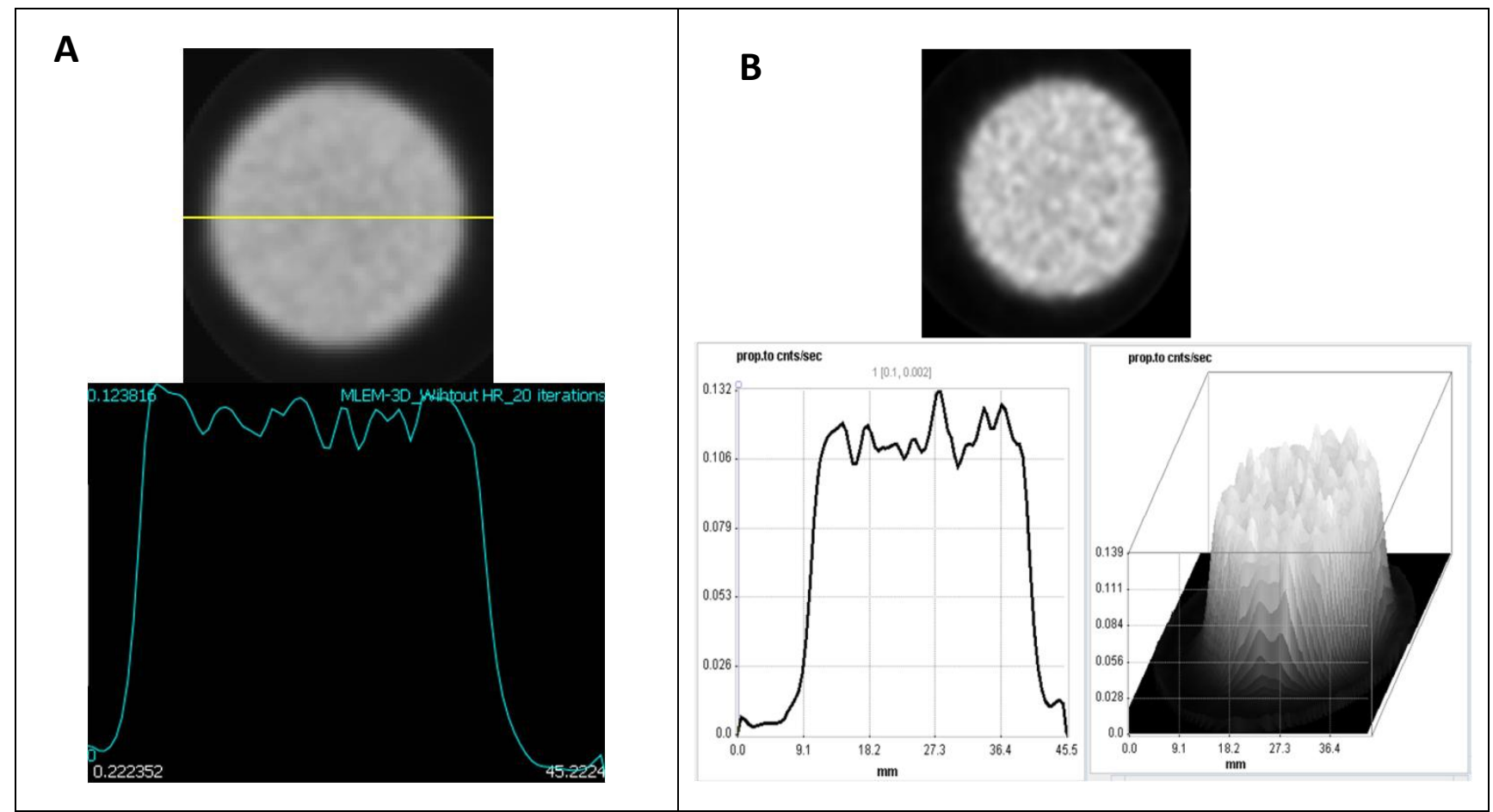


The mean, maximum and minimum activity concentration $(\mathrm{kBq} / \mathrm{mL})$ result and $(\% \mathrm{SD})$ obtained are presented in Table 3 for all evaluated reconstruction methods. Percentage standard deviation for all cases are also presented in Figure 4.

Table 3: $\%$ SD in uniform IQ Phantom Region using AMIDE and PMOD software.

\begin{tabular}{|c|c|c|c|c|c|c|}
\hline \multicolumn{7}{|c|}{ Uniformity } \\
\hline Software & $\begin{array}{c}\text { Reconstruction } \\
\text { Method } \\
\end{array}$ & HR * & $\begin{array}{c}\text { Mean } \\
(\mathrm{kBq} / \mathrm{mL}) \\
\end{array}$ & $\begin{array}{c}\text { Maximum } \\
(\mathrm{kBq} / \mathrm{mL})\end{array}$ & $\begin{array}{r}\text { Minimum } \\
(\mathrm{kBq} / \mathrm{mL})\end{array}$ & $\% \mathrm{SD}$ \\
\hline \multirow{5}{*}{ AMIDE } & FBP -2D & --- & 215,0 & 364,9 & 35,8 & 17,5 \\
\hline & \multirow{2}{*}{ MLEM-3D } & No & 187,2 & 238,5 & 142,2 & 6,7 \\
\hline & & Yes & 200,3 & 262,3 & 146,8 & 7,1 \\
\hline & \multirow{2}{*}{ OSEM-3D } & No & 195,8 & 249,4 & 146,7 & 6,7 \\
\hline & & Yes & 200,5 & 261,6 & 146,8 & 7,1 \\
\hline \multirow{5}{*}{ PMOD } & FBP -2D & --- & 194,9 & 350,9 & 34,4 & 17,6 \\
\hline & \multirow{2}{*}{ MLEM-3D } & No & 190,1 & 243,9 & 145,4 & 6,7 \\
\hline & & Yes & 197,8 & 262,8 & 147,0 & 7,1 \\
\hline & \multirow{2}{*}{ OSEM-3D } & No & 196,4 & 253,7 & 149,2 & 6,7 \\
\hline & & Yes & 197,1 & 262,5 & 146,9 & 7,1 \\
\hline
\end{tabular}

* High Resolution

Figure 4: \% SD in uniform IQ phantom region

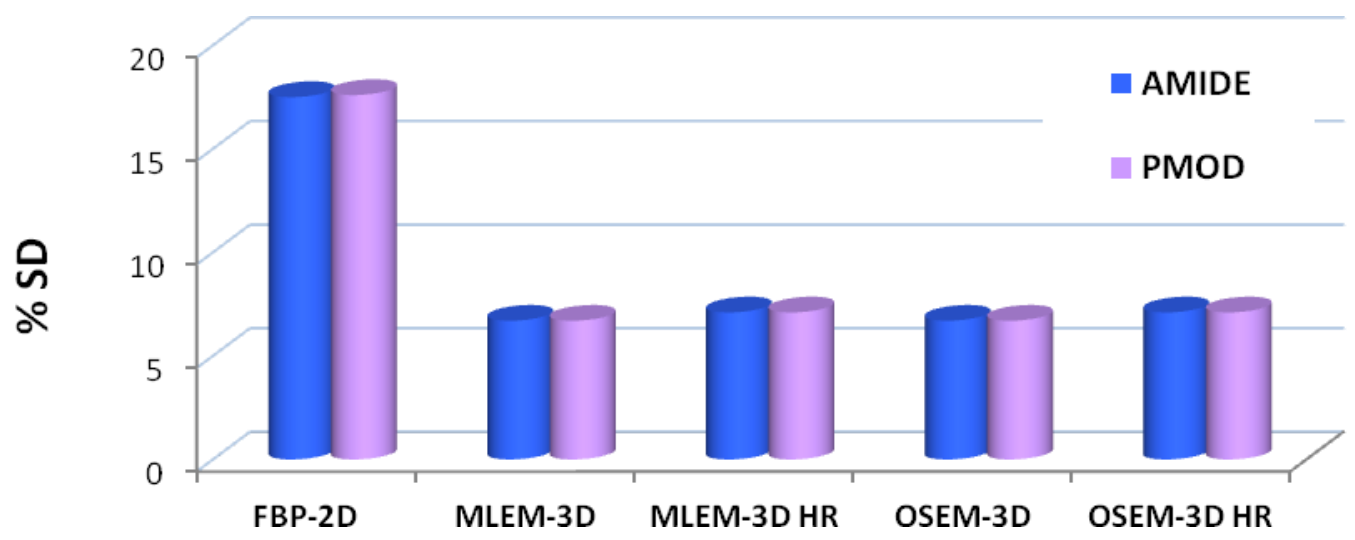


The analytic method presented highest standard deviation (FBP: 17,6\%SD) than iterative methods (MLEM without HR: 6,7\%STD; with HR: 7,1\%STD and OSEM without HR: 6,7\%STD; with HR: $7,1 \%$ STD). These values obtained for iterative methods are in accordance with results by Zaidi and Ratib [7] for LabPET 8TM.

\subsection{Recovery Coefficient}

Figure 5 presents a typical image of the 5 rods region.

Figure 5: Image of the IQ phantom showing a transverse plane of the 5 rods region.

A) AMIDE software. B) PMOD software

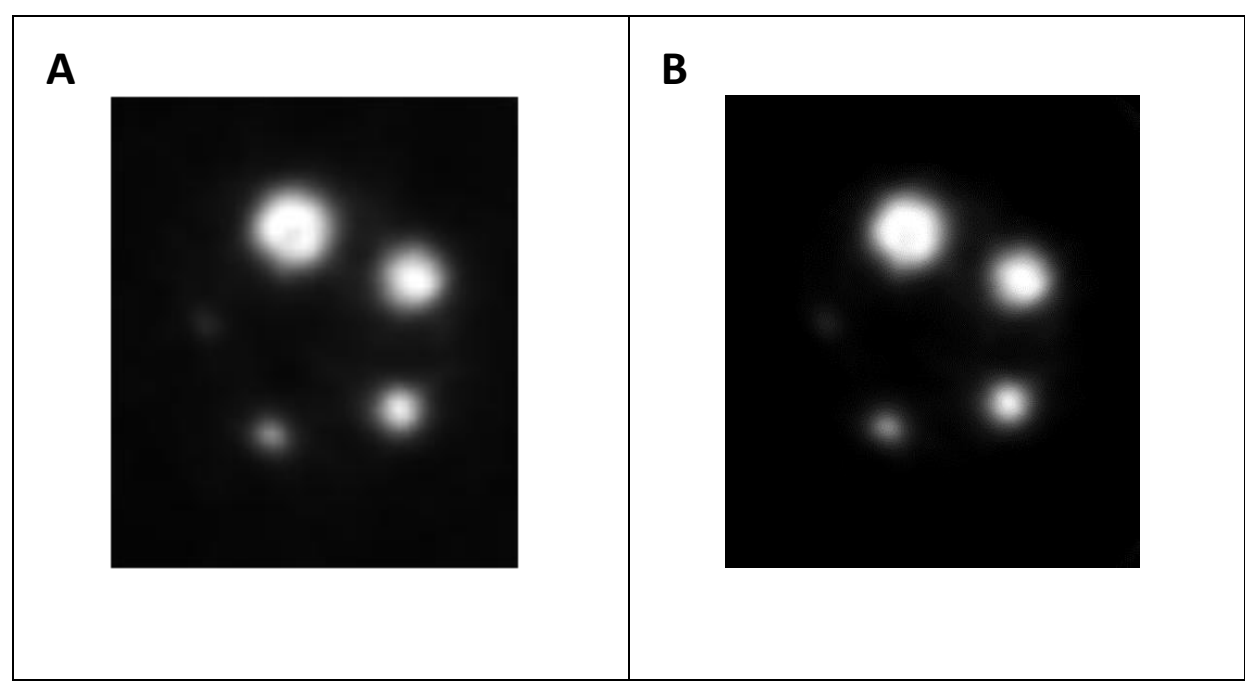

The RC's from the 5 rods ( 1 to $5 \mathrm{~mm}$ ) and respective standard deviations for different algorithms are given in Table 4. 
Table 4: RC's of different rods (1 to $5 \mathrm{~mm}$ ), reconstructed with distinct reconstruction algorithms.

\begin{tabular}{|c|c|c|c|c|c|c|c|c|c|c|c|c|}
\hline \multicolumn{13}{|c|}{ Recovery Coefficient (RC) } \\
\hline Software & $\begin{array}{c}\text { Reconstruction } \\
\text { Method } \\
\end{array}$ & HR & $1 \mathrm{~mm}$ & $\%$ STD & $2 \mathrm{~mm}$ & $\%$ STD & $3 \mathrm{~mm}$ & $\%$ STD & $4 \mathrm{~mm}$ & $\%$ STD & $5 \mathrm{~mm}$ & $\%$ STD \\
\hline \multirow{5}{*}{ AMIDE } & FBP -2D & --- & 0,17 & 66,0 & 0,38 & 60,9 & 0,38 & 59,2 & 0,78 & 59,1 & 0,86 & 58,6 \\
\hline & \multirow{2}{*}{ MLEM-3D } & No & 0,12 & 28,4 & 0,43 & 27,2 & 0,77 & 26,5 & 0,82 & 25,5 & 0,87 & 26,3 \\
\hline & & Yes & 0,13 & 27,1 & 0,47 & 22,0 & 0,80 & 22,6 & 0,88 & 21,5 & 0,85 & 23,0 \\
\hline & \multirow{2}{*}{ OSEM-3D } & No & 0,12 & 28,7 & 0,43 & 22,5 & 0,79 & 22,4 & 0,87 & 22,4 & 0,85 & 22,2 \\
\hline & & Yes & 0,13 & 27,5 & 0,46 & 20,3 & 0,80 & 21,0 & 0,89 & 20,4 & 0,87 & 21,4 \\
\hline \multirow{5}{*}{ PMOD } & FBP -2D & --- & 0,17 & 60,2 & 0,38 & 54,0 & 0,62 & 52,3 & 0,79 & 52,0 & 0,87 & 51,7 \\
\hline & \multirow{2}{*}{ MLEM-3D } & No & 0,12 & 31,0 & 0,48 & 27,7 & 0,79 & 28,0 & 0,86 & 27,9 & 0,87 & 27,9 \\
\hline & & Yes & 0,13 & 33,5 & 0,47 & 28,5 & 0,80 & 29,6 & 0,87 & 28,4 & 0,87 & 29,3 \\
\hline & \multirow{2}{*}{ OSEM-3D } & No & 0,12 & 31,2 & 0,44 & 27,5 & 0,78 & 28,0 & 0,86 & 28,2 & 0,86 & 27,2 \\
\hline & & Yes & 0,13 & 34,1 & 0,46 & 30,3 & 0,79 & 31,3 & 0,87 & 30,5 & 0,87 & 31,0 \\
\hline
\end{tabular}

The RCs of the rods for all reconstruction methods in both softwares are shown in Figure 6.

Figure 6: $R C$ s of different rods (1 to $5 \mathrm{~mm}$ ), reconstructed with distinct reconstruction methods.

A) AMIDE software. B) PMOD software.
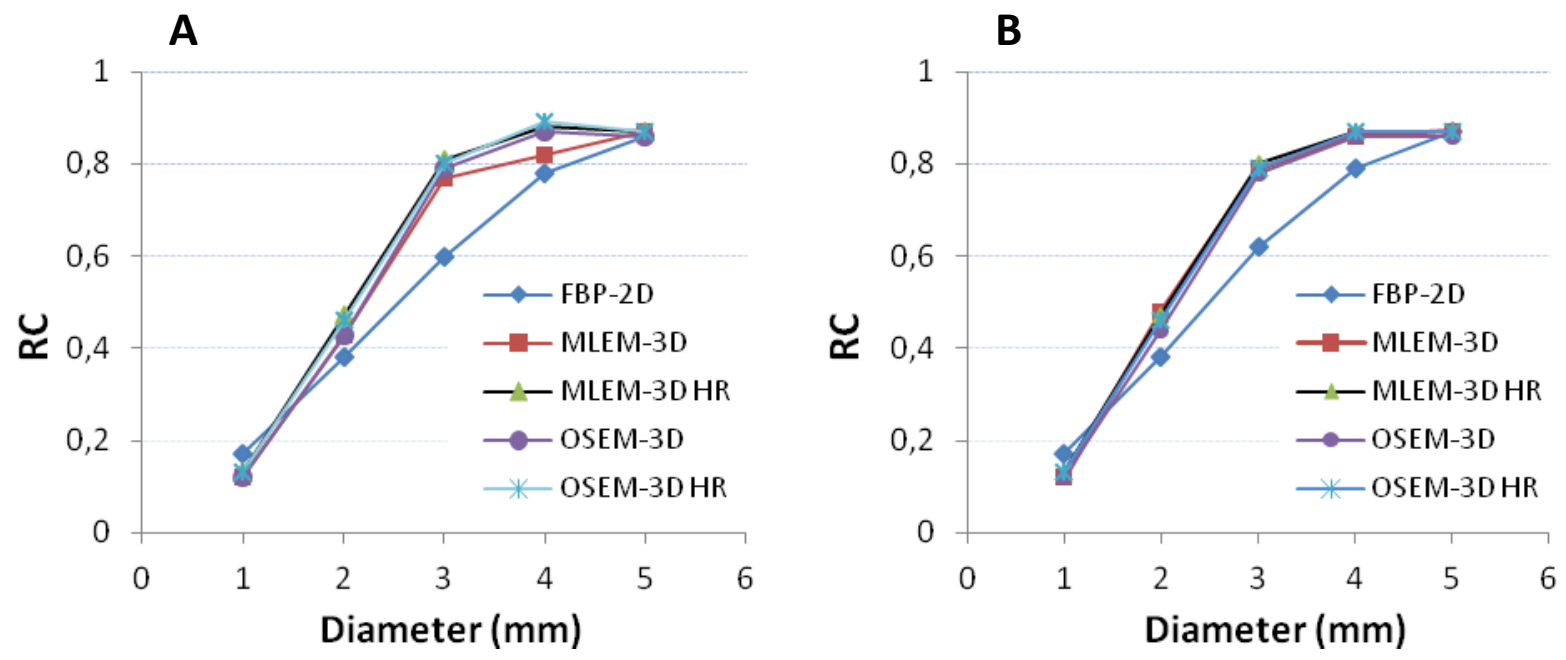
In the above results, it can be noticed that the RC values for the 5 different diameters ranged from 0.12 to 0.87 and are close to each other in both iterative reconstruction methods (MLEM and OSEM) adopted and software used. The RCs using FBP method ranged from 0.17 to 0.87 in both software used.

The standard deviation increases as the rod diameter decreases in all cases studied. These values are similar to those reported earlier for the LabPET ${ }^{\mathrm{TM}}$ scanner [7].

\subsection{Spillover Ratio}

Figure 7 presents a typical image of the 2 non-radioactive compartments region.

Figure 7: Image of the IQ phantom showing a transverse plane of the 2 non-radioactive compartments region. A) AMIDE software. B) PMOD software.

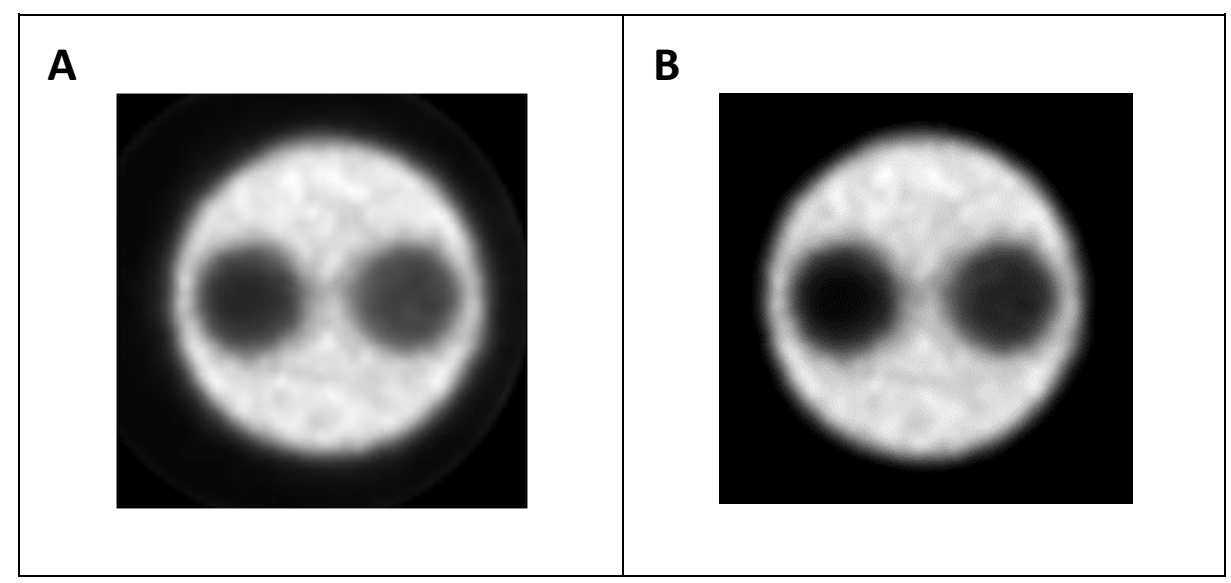

The SOR's and respective \% SD in the water and air compartments are reported in Table 5. 
Table 5: SORs and \%STD in water and air, reconstructed with distinct reconstruction algorithms.

\begin{tabular}{|c|c|c|c|c|c|c|}
\hline \multirow{3}{*}{ Software } & \multicolumn{6}{|c|}{ Spillover Ratio (SOR) } \\
\hline & \multirow{2}{*}{ Reconstruction Method } & \multirow{2}{*}{ HR } & \multicolumn{2}{|c|}{ Water } & \multicolumn{2}{|c|}{ Air } \\
\hline & & & SOR & $\%$ STD & SOR & $\%$ STD \\
\hline \multirow{5}{*}{ AMIDE } & FBP -2D & --- & 0,07 & 243,1 & 0,26 & 72,3 \\
\hline & \multirow{2}{*}{ MLEM-3D } & No & 0,18 & 14,1 & 0,29 & 12,4 \\
\hline & & Yes & 0,19 & 13,1 & 0,29 & 13,1 \\
\hline & \multirow{2}{*}{ OSEM-3D } & No & 0,18 & 14,5 & 0,29 & 12,6 \\
\hline & & Yes & 0,18 & 14,9 & 0,29 & 13,4 \\
\hline \multirow{5}{*}{ PMOD } & FBP -2D & --- & 0,08 & 189,7 & 0,27 & 61,3 \\
\hline & \multirow{2}{*}{ MLEM-3D } & No & 0,18 & 13,2 & 0,29 & 12,2 \\
\hline & & Yes & 0,18 & 13,5 & 0,29 & 12,8 \\
\hline & \multirow{2}{*}{ OSEM-3D } & No & 0,18 & 13,8 & 0,29 & 12,0 \\
\hline & & Yes & 0,18 & 13,8 & 0,30 & 12,7 \\
\hline
\end{tabular}

The SOR's of the water and air compartments are shown in Figure 8. 
Figure 8: SORs of different compartments (water and air), reconstructed with distinct reconstruction methods. UPPER: AMIDE software. BOTTOM: PMOD software.
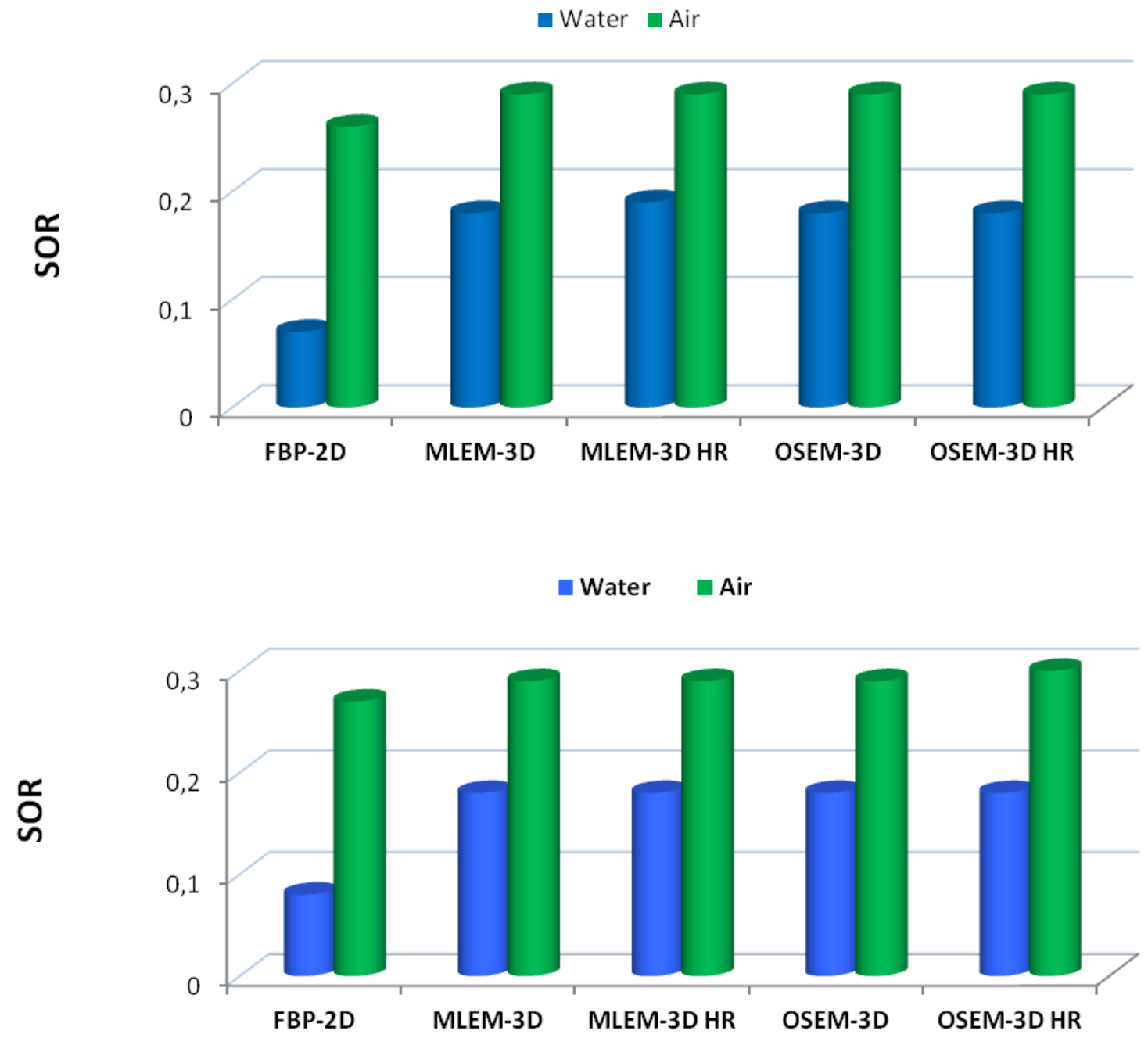

The accuracy of scatter corrections is given as the spillover ratio in cold areas. Values of this work are comparable those from Zaidi and Ratib work [7].

\section{CONCLUSION}

The results obtained in this work using IQ phantom defined by NEMA NU 4/2008 reveals that the system LabPET 4 of LIM/CDTN/CNEN produces images with performance according to the litera- 
ture. The present study is an initial step to verify the NEMA NU 4/2008 for further standardization. Next steps will be to implement the others sections of the NEMA NU 4/2008 Standard in the LIM/CDTN.

\section{ACKNOWLEDGMENT}

The authors would like to thank the staff of the LIM and UPPR-CDTN/CNEN. This work was supported and financed by FAPEMIG, UFMG and CDTN.

\section{REFERENCES}

1. YAO R.; LECOMTE R.; CRAWFORD E. Small-Animal PET: What is it, and why do we need it? Journal of Nuclear Medicine Technology. 2011. Vol. 40 n³, pp.157-165.

2. NEMA - National Electrical Manufacturers Association. Performance Measurements of Small Animal Positron Emission Tomographs. Rosslyn VA; 2008 Standards Publication NU 4-2008.

3. GE Healthcare Technologies, Triumph Service Guide Technical Publication. Revision Draft 6, Copyright. Available at: <http://www.gehealthcare.com>. 2011.

4. TRETRAULT M. A., et al. System architecture of the LabPET small animal PET scanner. IEEE Transactions on Nuclear Science, 2008. Vol.55, pp. 2546-2550.

5. FONTAINE R., et al. The hardware and signal processing architecture of LabPET ${ }^{\mathrm{TM}}$, a small animal APD-based digital PET scanner. IEEE Transactions on Nuclear Science, February 2009. Vol. 56, pp. 3-9.

6. DISSELHORST J. A., et al. Image-Quality Assessment for several Positron Emitters using the NEMA NU 4-2008 Standards in the Siemens Inveon Small-Animal PET Scanner. The Journal of Nuclear Medicine. April 2010. Vol. $51 \mathrm{n}^{\circ} 4$, pp. 610-617.

7. ZAIDI H. and RATIB O. NEMA NU-04-based Performance Characteristics of the LabPET8TM Small Animal PET Scanner. 2001. IEEE Nuclear Science Symposium Conference Record. pp. 3654-3660. 